\title{
ELECTRIC POLARIZATION OF ONSAGER FLUIDS. II. BIREFRINGENCE. 3. ROLE OF UNIVERSAL PAIRWISE INTERACTIONS
}

\author{
V.V. Prezhdo ${ }^{a}$, G.V. Tarasova ${ }^{b}$, O.V. Prezhdo ${ }^{c}$, S.A. Tyurin ${ }^{b}$, \\ O.N. AKULOVA ${ }^{b}$ AND N.I. IVANOV ${ }^{b}$ \\ ${ }^{a}$ Institute of Chemistry, University of Opole, Oleska 48, 45-951 Opole, Poland \\ ${ }^{b}$ Ukrainian Scientific Center for Water Control, Kharkov, Ukraine \\ ${ }^{c}$ Department of Chemistry, University of Texas, Austin, USA
}

(Received August 10, 1995)

\begin{abstract}
A comparative analysis of several methods of interpretation of solvent influence on molar Kerr constants of solutes was done. The analysis was based on experimentally determined electro-optical and dielectric properties of binary solutions of organic substances with various polarities, polarizabilities and optical anisotropies. It was found that the role of universal van-der-Waals interactions treated via London-Debye-Keesom potentials was best accounted for by a modified orientational theory of Kerr effect that developed the ansatz of reactive field and local dielectric permittivity. An extrapolation method of determination of gas phase molar Kerr constants of solutes was deduced from the theory.
\end{abstract}

PACS numbers: $31.70 . \mathrm{Dk}, 78.20 . \mathrm{Fm}, 72.80 . \mathrm{Jc}$

\section{Introduction}

Molecular polarizability ellipsoid is traditionally obtained from the three fundamental relationships between experimental data on molecular refraction, Rayleigh light scattering, and Kerr effect and the components of the second range tensor of molecular polarizability. Electric birefringence (EB, Kerr effect) constants reflect electric and polarizational properties of particles. They range in more than seven orders of magnitude for qualitatively different substances and thermodynamic states of matter. It is quite remarkable for a directly measurable property and leads to a very high sensitivity of Kerr effect measurements to slight changes in structural and physical-chemical properties of substances, and in particular energy changes brought by various types of interparticle interactions. 


\section{Theoretical description of Kerr effect in solutions}

Molar Kerr constant is one of the basic parameters of EB. It is an intensive characteristic of matter which relates experimentally measured birefringence of an electrically polarized anisotropic medium with the microscopic properties of particles - electric $\left(\alpha_{i j}^{\mathrm{e}}\right)$ and optical $\left(\alpha_{i j}^{\circ}\right)$ polarizability tensors and dipole moment vector $\left(\mu_{i}\right)$. According to the classic Langevin-Born theory of EB of gases the relationship can be written as

$$
\begin{aligned}
& m K^{\mathrm{g}}=K^{\mathrm{g}} N^{-1}=3 \pi N_{\mathrm{A}}\left(\Theta_{1}^{\mathrm{g}}+\Theta_{2}^{\mathrm{g}}\right), \\
& \Theta_{1}^{\mathrm{g}}=2\left(D^{\mathrm{e}}: D^{\circ}\right)\left(15 k_{\mathrm{B}} T\right)^{-1}, \Theta_{2}^{\mathrm{g}}=\left(D^{\mathrm{e}}: \mu_{i} \cdot \mu_{j}\right)\left(45 k_{\mathrm{B}}^{2} T^{2}\right)^{-1},
\end{aligned}
$$

where $K^{\mathrm{g}}$ is measured birefringence of a gas containing $N$ particles in a unit volume, $N_{\mathrm{A}}$ and $k_{\mathrm{B}}$ are Avogadro and Boltzmann constants, $\Theta_{1}^{\mathrm{g}}$ and $\Theta_{2}^{\mathrm{g}}$ are anisotropic and dipole contributions to molar Kerr constant $m K^{\mathrm{g}}, D^{\mathrm{e}}$ and $D^{\circ}$ are deviators of particles electric and optical polarizability tensors at absolute temperature $T$. Development of a corresponding theory for condensed phases is impeded by several factors with the most important ones being the necessity to account for intermolecular interactions (IMI) influence on experimental EB parameters, Joule effect, nonlinearity of polarizabilities, and difference between the acting (internal) and applied (external) electric fields. Statistical and quantum mechanical theories which consider the above mentioned effects together with interparticle spatial and angular correlations use complicated models involving parameters which are difficult or impossible to measure or compute. Application of such theories is severely limited to the simplest cases. At the same time there exists a number of analytical expressions which allow for calculation of molar Kerr constants of solutes in liquids $\left(m K^{l}\right)$ from experimental data on EB and dielectric constants. These expressions are derived on the basis of experimental characteristics of condensates interpreted using the traditional Langevin-Born theory of gas phase EB or its modifications. Modifications are usually obtained by varying description of either internal field or Lorentz function. Review on the subject can be found in [1]. Staying within the limits of this approach we introduced a new internal field model [2] developing the concepts of reactive field and local electric induction. Later, with the help of the model we developed a theory of EB of molecular condensates [3]. In particular, we derived

$$
\begin{aligned}
m K^{l} & =3 K^{l} M n^{2}(1-\alpha t) g\left(n^{2}+2\right) \rho^{-1}=3 \pi N_{\mathrm{A}}\left(\Theta_{1}^{l}+\Theta_{2}^{l}\right) \\
& =m K^{\mathrm{g}}+15 \pi N_{\mathrm{A}} \alpha t(1+0.25 \alpha t)(1-\alpha t)^{2} \Theta_{2}^{\mathrm{g}}
\end{aligned}
$$

where $K^{\mathrm{g}}$ is an observed birefringence of molecules with dynamic polarizability $\alpha$; $M, n$, and $\rho$ are molecular weight, refraction index and density of the medium; $g$ and $t$ are cavity and reactive internal field factors [4]. Reports on experimental studies of binary solutions with inert, apolar solvents, which do not undergo complex formation, comprise a major part of literature data on molar Kerr constants. A corresponding equation allowing to obtain molar Kerr constants ${ }_{\infty}\left(m K_{2}^{l}\right)$, which are the best approximations to the gaseous phase values [2], on the basis of EB and dielectric constant data for such solutions has the following form [5]: 


$$
\begin{aligned}
& \infty\left(m K_{\mathrm{g}}^{l}\right)=\frac{3 M_{2}\left(1-\alpha_{2} t_{1}\right) n_{1}^{2}}{g_{1} \rho_{1}\left(n_{1}^{2}+2\right)} K_{1}^{l}[1+\xi-\beta \\
& \left.\quad-\left(\frac{\mathrm{d}}{\mathrm{d} x_{2}} \ln g_{0}\right)_{x_{2}=0}-\frac{1}{1-\alpha_{1} t_{1}}\left(\frac{\mathrm{d}}{\mathrm{d} x_{2}} \alpha_{1} t_{0}\right)_{x_{2}=0}+\frac{4 \gamma}{n_{1}^{2}+2}\right] .
\end{aligned}
$$

Here we assume and experimentally prove that the following correlations hold:

$$
\begin{array}{ll}
K_{0}^{l}=K_{1}^{l}\left(1+\xi x_{2}\right), & \rho_{0}=\rho_{1}\left(1+\beta x_{2}\right), \\
n_{0}=n_{1}\left(1+\gamma x_{2}\right), & \varepsilon_{00}=\varepsilon_{01}\left(1+\delta x_{2}\right) .
\end{array}
$$

Here, $\varepsilon_{0}$ is static dielectric permittivity of the medium. Indices $i=0,1,2$ denote solution, solvent and solute correspondingly, $x_{2}$ is mole fraction of solute. It is significant that $\infty\left(m K_{2}^{l}\right)$ of Eq. (3) depends not only on solute and solvent properties, but also on properties of solution per se. It is clear that EB constants of solute molecules interacting with a solvent depend on physical-chemical characteristics of the latter (so-called solvent effect). Solvent influence on $m K_{2}^{l}$ has been investigated by several researches [6]. A number of empirical equations relating molecular properties of individual substances with macroscopic parametcrs of solution has been suggested. Thus, the authors of [7] studied the correlation between molar constants of apolar solutes with a single functional group dissolved in apolar solvents and Lorentz-Lorenz functions. They showed that $m K^{\mathrm{g}}$ could be reasonably well reproduced by extrapolating the correlation to $n=1$. However, such correlation is not quite valid, mainly because refraction index $n$ is a function of the trace of polarizability tensor $A_{i j}$ and not of its deviator.

Paper [8] experimentally established that the ratio of $\infty_{\infty}\left(m K_{2}^{l}\right)$ of substances dissolved in polar solvents is proportional to the ratio of dipole moments of the substances squared. Another paper [9] gave a theoretical footing for this result which can hold only for compounds with large values of $\Theta_{2}^{\mathrm{g}}$ of Eq. (1) and provided that molecular dipole moment is collinear with the main axis of polarizability ellipsoid. For some substances the correlation breaks down when they exhibit specific IMI with solvents. In particular, the ratio of $\infty\left(m K_{2}^{l}\right)$ of nitro and chlorophenols dissolved in dioxane and toluene is almost an order of magnitude greater than the ratio of squares of their dipole moments [10]. It happens because the phenols form stable complexes with dioxane and toluene molecules. The above discussion together with the critical review of approaches to the modeling of the relationship between $m K_{2}^{l}$ values and solvent effect parameters [6] evidence that the major causes of the solvent effect - van-der-Waals and specific IMI - are not fully accounted for by these approaches.

At the same time a good description of IMI is an important factor for successful estimation of gas phase molar Kerr constants of solutes. For example, a solute molecule in an apolar solvent is surrounded by a solvation shell containing solvent molecules with a somewhat preferred orientation due to dipole-induced dipole and dispersive IMI. Such arrangement interferes with the spatial dynamics of the solute molecule acted upon by an external electric field. Solute molecule dynamics becomes correlated with dynamics of the solvation shell molecules leading to changes in optical polarizability tensor and, according to the orientational theory of Kerr effect and Eq. (1), to changes in EB constants. 
Determination of potential energy of interacting particles is a complicated many-body problem which usually cannot be solved exactly. A semi-empirical approach, on the other hand, uses many microscopic parameters specific to a particular molecular condensate. For these reasons analytic expressions derived within such formalisms cannot be applied to a majority of experimental data. The most popular way to avoid the difficulty is to mimic interactions by independent pairwise electrostatic potentials. In such case the total energy is just a sum of energies of interacting pairs of point charges. These empirical potentials are equivalent to averaged quantum mechanical potentials obtained via multipole expansion and perturbation theory under the assumption that charge distributions of different particles do not overlap.

Potential energy of van-der-Waals IMI in dense molecular media, and in solutions in particular, is a function of properties of individual molecules and molecular structure of a medium as a whole. According to [11] the potential energy can be well approximated by the energy of pairwise long range forces depending on polarizability and polarity of molecules. Accidentally, polarizability and polarity also determine EB constants and play the major role in the orientational theory of Kerr effect (1). Following [12] we express IMI between solutes and apolar solvents in terms of the corresponding London $\left(\varphi_{\mathrm{L}}\right)$ and Debye $\left(\varphi_{\mathrm{D}}\right)$ potentials (LD potentials). Configurationally averaged $(\tau)$ energy of pairwise non-specific interactions between molecules in a binary solution is taken as

$$
\begin{aligned}
& \left\langle U_{12}\right\rangle_{\tau}=\sum_{m}\left(\frac{Z}{\bar{R}^{6}}\right)_{m}\left\langle\delta_{\text {disp }} \varphi_{\mathrm{L}}+\delta_{\text {ind }} \varphi_{\mathrm{D}}\right\rangle_{\tau} \\
& =A \sum_{m}\left(\frac{Z}{\bar{R}^{6}}\right)_{m} \alpha_{1}\left(\frac{1.5 I_{11} I_{12}}{I_{11}+I_{12}} \alpha_{2}+\mu_{02}^{2}\right)=A \sum\left(\frac{Z}{\bar{R}^{6}}\right)_{m} \alpha_{1} B_{12}(I, \alpha, \mu), \\
& Z_{m}=\frac{N_{\mathrm{A}}}{V}\left(\frac{4 \pi}{3} \bar{R}_{m}^{3}-V_{m}\right), \quad V_{m}=\sum V_{\mathrm{at}}, \\
& \bar{R}_{m}=R_{2}+(2 m-1) R_{1}, \quad R_{i}=\left(4 \pi b M_{i} / 3 \rho_{i} N_{\mathrm{A}}\right)^{1 / 3},
\end{aligned}
$$

where $Z_{m}$ is a number of molecules in the $m$-th solvation shell of radius $R_{m}, A$ is a parameter accommodating all non-additivity corrections $\delta_{j}$ to LD potentials $\varphi_{j} ; I_{1 j}$ and $\alpha_{i}$ are the first ionization potentials and dynamical polarizabilities of solvent $(i=1)$ and solute $(i=2)$ molecules; $V, V_{m}$, and $V_{\text {at }}$ are molar, molecular, and atomic volumes; $R_{i}$ denotes radius of a spherical cavity occupied by a solute molecule; $b$ is packing factor. A logical assumption of proportionality of changes of all molecular properties of solutes including electro-optical ones during the transition from a gas phase to a binary solution can be written for the first" solvation shell $(m=1, b=0.74)$ as

$$
\left|m K_{2}^{\mathrm{g}}-m K_{2}^{\prime}\right| \approx B_{12}(I, \alpha, \mu) \frac{Z_{1}}{\bar{R}^{6}} \alpha_{1}=B_{12} Z_{\alpha} .
$$

Definition of the IMI potential energy by a function of statistically averaged numbers of particles within solvation shells and average distances between interacting solute and solvent molecules for each shell (Eq. (5)) is more coherent with the modern theories of molecular condensates than the corresponding expression of 
continuum models. Another advantageous feature of Eq. (5) stems from the fact that $\left\langle U_{12}\right\rangle_{\tau}$ explicitly depends not only on solute and solvent parameters, but also on the properties of the entire solution. All of the above mentioned definitely helps to achieve our goal: to rationalize IMI influence on electro-optical properties of solutes by systematically varying physical-chemical, structural and other properties of solutes and solvents.

In Monte Carlo (MC) and molecular dynamics (MD) simulations potential energy of a solute molecule in an apolar solvent is determined by its IMI with solvent molecules situated within a cut-off radius $R_{c}$. The total energy of (5) can be evaluated from the first principles of quantum mechanics applied to a many-body system consisting of molecules within a sphere of radius $R_{c}$. However, the radius of the approximately spherical solvation shell defined by continuum theories of liquids from polarizations and inductances if solution components and dipole screening constants significantly exceeds $R_{\mathrm{c}}$. I follows, for instance, from the structural analysis of dilute binary solutions and data on amplitudes of various radial distribution functions [13]. To keep our approach consistent (within the limits of the considered problem only!) with MC and MD methods of calculation of dielectric properties of molecular condensates we express the total energy of (5) as

$$
U_{\Sigma}=\frac{1}{2} \sum_{q} U_{p q}-U_{R p}=\frac{1}{2} \sum_{q} U_{p q}-\frac{\varepsilon_{\mathrm{c}}-1}{2 \varepsilon_{\mathrm{c}}+1} \frac{M_{\Sigma} \mu_{p}}{R_{c}^{6}},
$$

where $U_{p q}$ is energy of a solvent molecule $p$ with dipole moment $\mu_{p}$ due to IMI described via LD potentials. The molecule is situated in the center of a sphere of radius $R_{\mathrm{c}}$ filled with solvent particles $(q)$. Dense van-der-Waals packing is assumed; $\varepsilon_{\mathrm{c}}$ is dielectric constant of solution; $U_{R p}$ denotes energy of dipole $\mu_{p}$ placed in reactive field $M_{\Sigma}=M\left(E_{\mu}, \tau\right)$ generated by solvation shell molecules charge densities fluctuating under the influence of the instantaneous dipole $\mu_{p}$. We estimated magnitudes of both terms of (7) using the statistical technique of [14] to calculate $M_{\Sigma}$ and found that $U_{R p}$ contributes up to twenty percent to the total energy.

Our preliminary analysis [10] of reference data [15] on molar Kerr constants of gaseous organic substances and those dissolved in apolar solvents showed that the following correlations corresponding to Eq. (7) satisfactorily hold for the studied ranges of $Z_{1}$ and $\alpha_{1}$ values:

$$
m K_{2}^{l},\left|m K_{2}^{\mathrm{g}}-m K_{2}^{l}\right| \approx \text { const }+B_{12} Z_{\alpha} .
$$

It is important to note that our approach relating measured EB constants with pairwise IMI energies can succeed only if experimental and theoretical characteristics of EB are correctly determined. We meet the requirement on experimental data by using the compensatory method [6] of measurement of EB of condensed media affected by a low frequency alternating polarizing electric field. Agreement between the calculated values of $\infty_{\infty}\left(m K_{2}^{l}\right)$ and the corresponding gas phase values is the main test of the theory. The basic idca of our approach to systematically account for reactive field contribution to the total potential energy of a medium held under EB conditions by substituting Lorentz field by Onsager field or its modifications has been checked against experimental and theoretical data on organic compounds characterized by a wide range of polarizational and structural properties $[3,16]$. 


\section{Results and discussion}

We measured dielectric and electro-optical properties of a series of dilute binary solutions of organic compounds with concentrations satisfying conditions (4). The technique and equipment are described elsewhere [2,5]. The solvents were alkanes and their halogene and sulphur derivatives, cycloalkanes, aromatic hydrocarbons and cyclic oxides. All of them are apolar substances with similar ionization potentials $\left(I_{11}\right)$ and polarizabilities $\left(\alpha_{1}\right)$. Their physical properties are given in Table I, where $\sigma$ denotes Lennard-Jones scaling parameter, $\omega$ is Pitzer's asphericity parameter [17], $\bar{\alpha}$ is an average static molecular polarizability, $\gamma_{l}^{2}$ denotes molecular anisotropy [15] and $I_{1}$ is the first ionization potential [18]. The solutes were polar and apolar representatives of alkanes, sulphur and halogene derivatives of alkanes, benzene and its alkyl, amino, and nitro derivatives, other aromatic hydrocarbons, ethers, ketones, derivatives of carbonic acids (Table II). To compute molar Kerr constants $\infty\left(m K_{2}^{l}\right)_{e}$ we used several schemes including orientational theory of EB with Onsager $(l=1)[19]$ and Block-Walker $(l=2)$ [20] internal field models and Langevin-Born theory $(l=3)$ Eq. (3) modified to treat dilute binary solutions. The latter is based on the quasi-continuum internal field model developed earlier [2]. The results are given in Tables I, II and are partially depicted in Figs. 1, 2. The data can be interpreted as fol-

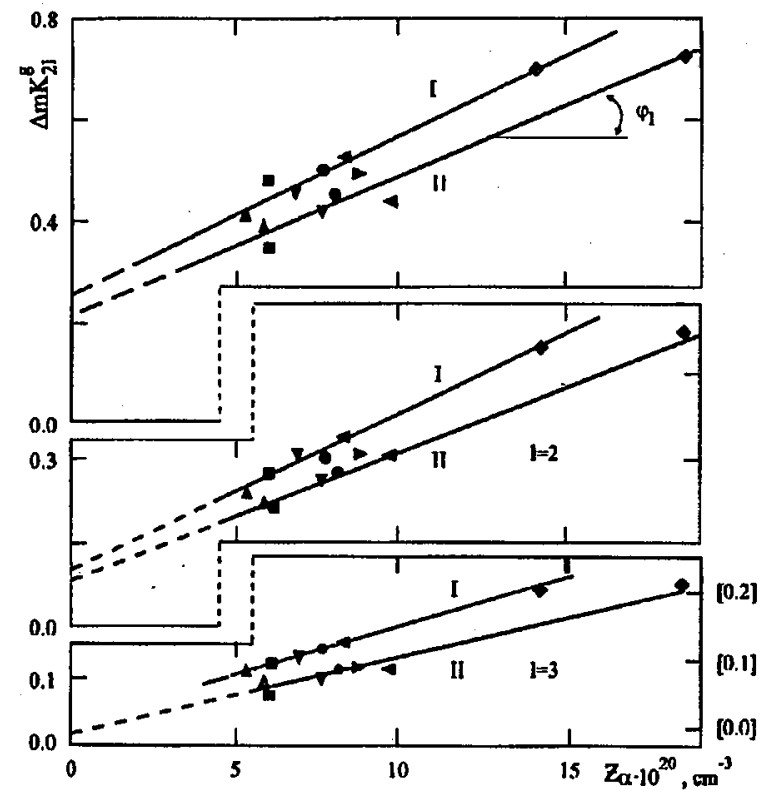

Fig. 1. The dependence of $\Delta m K_{2 l}^{\mathrm{g}}$ of $Z_{\alpha}$ parameter for: naphtalene (I) and benzene (II).

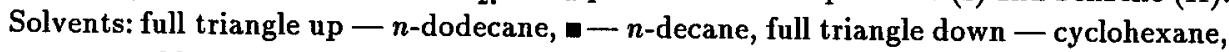
- - tetrachloromethane, full triangle left - benzene, full diamond - carbon disulfide. 


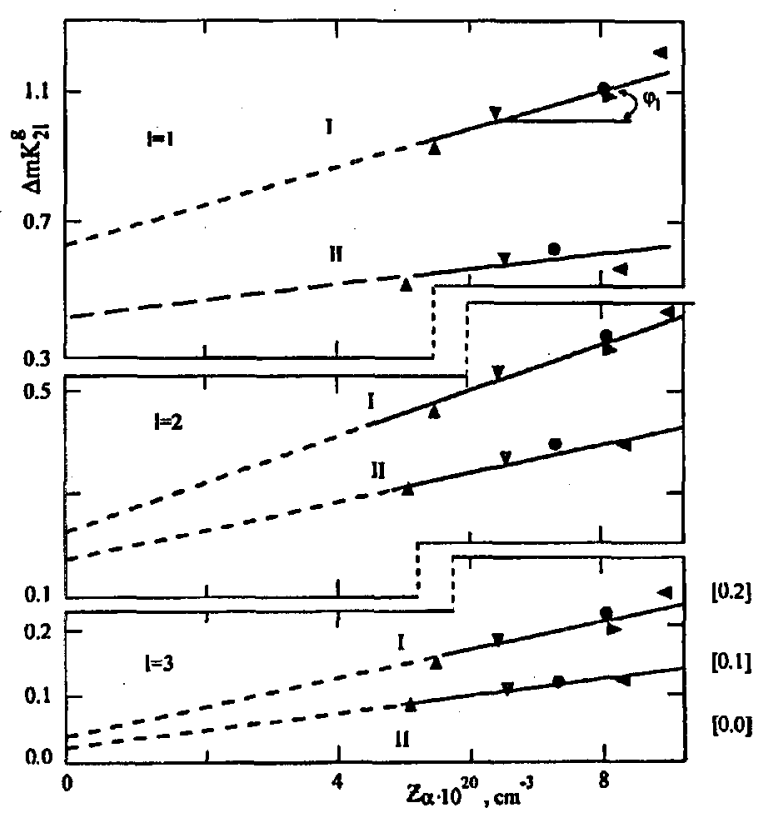

Fig. 2. The dependence of $\Delta m K_{2 l}^{\mathrm{g}}$ of $Z_{\alpha}$ parameter for: nitrobenzene (I) and naphtalene (II). Solvents: full triangle up $-n$-heptane, full triangle down - cyclohexane, $\bullet-$ tetrachloromethane, full triangle right - 1,4-dioxane, full triangle left - benzene, full diamond - carbon disulfide.

TABLE I

The properties of the apolar solvents.

\begin{tabular}{c|l|c|c|r|r|r}
\hline \hline No. & \multicolumn{1}{|c|}{ Solvent } & $\sigma[\AA]$ & $\omega$ & $\bar{\alpha}\left[\AA^{3}\right]$ & $\gamma_{l}^{2}\left[\AA^{6}\right]$ & $I_{1}[\mathrm{eV}]$ \\
\hline 1 & $n$-heptane & 5.561 & 0.351 & 13.67 & 6.1 & 10.08 \\
2 & $n$-hexane & 4.424 & 0.296 & 11.81 & 4.7 & 10.18 \\
3 & cyclohexane & 5.193 & 0.213 & 10.89 & 4.6 & 9.83 \\
4 & tetrachloromethane & 5.054 & 0.194 & 11.15 & - & 11.47 \\
5 & 1,4-dioxane & 4.737 & 0.288 & 10.17 & 2.8 & 9.13 \\
6 & benzene & 4.917 & 0.212 & 10.54 & 30.5 & 9.25 \\
7 & carbide disulfide & 4.329 & 0.115 & 8.44 & 76.1 & 10.07
\end{tabular}

lows. First of all, relationship (8) for $\Delta m K_{2 l}^{\mathrm{g}}\left(Z_{\alpha}\right)$ holds for significant ranges of molecular dipoles, polarizabilities and polarizability anisotropies of both solutes and solvents independent of a chosen theoretical model of Kerr effect and internal field. Pairwise molecular interactions are clearly of non-local nature for a large spectrum of structural characteristics of solution components. Thus, for example, calculated values of $\Delta m K_{2 l}^{\mathrm{g}}$ for both $\mathrm{CS}_{2}$ with $\omega=0.115$ and $n$ - $\mathrm{C}_{8} \mathrm{H}_{18}$ 
TABLE II

The molecular properties of the solutes.

\begin{tabular}{|c|c|c|c|c|c|c|c|}
\hline No. & Solvent & $\mu[\mathrm{D}]$ & $\omega$ & $\bar{\alpha}\left[\AA^{3}\right]$ & $\gamma_{c}^{2}\left[\AA^{6}\right]$ & $\begin{array}{c}m K \cdot 10^{12} \\
\text { CGSE }\end{array}$ & $\begin{array}{c}m K \cdot 10^{12} \\
{[15]}\end{array}$ \\
\hline 1 & $\mathrm{CS}_{2}$ & 0.0 & 0.115 & 8.44 & 76.1 & - & 36.76 \\
\hline 2 & $\mathrm{C}_{6} \mathrm{H}_{6}$ & 0.0 & 0.212 & 10.54 & 30.5 & 17.16 & 17.43 \\
\hline 3 & $\mathrm{CCl}_{4}$ & 0.0 & 0.194 & 11.15 & - & - & 0.51 \\
\hline 4 & $\mathrm{C}_{10} \mathrm{H}_{8}$ & 0.0 & 0.394 & 16.04 & 6.4 & - & 9.64 \\
\hline 5 & $\mathrm{C}_{10} \mathrm{H}_{8}$ & 0.0 & 0.302 & 17.65 & 219.3 & - & 40.81 \\
\hline 6 & $\mathrm{C}_{14} \mathrm{II}_{10}$ & 0.0 & - & - & 430.7 & - & 102.15 \\
\hline 7 & $1,4-\left(\mathrm{NO}_{2}\right)_{2} \mathrm{C}_{6} \mathrm{H}_{4}$ & 0.17 & 0.324 & 14.53 & 78.6 & - & 31.10 \\
\hline 8 & $\left(\mathrm{C}_{6} \mathrm{H}_{5}\right)_{3} \mathrm{CH}$ & ن.19 & - & 31.09 & 52.5 & - & 13.18 \\
\hline 9 & $\mathrm{C}_{6} \mathrm{H}_{5} \mathrm{CH}_{3}$ & 0.34 & 0.257 & 13.28 & 47.0 & 33.06 & 32.13 \\
\hline 10 & $\mathrm{CHCl}_{3}$ & 1.01 & 0.216 & 17.84 & 9.3 & -29.12 & -30.02 \\
\hline 11 & $\left(\mathrm{C}_{2} \mathrm{H}_{5}\right)_{2} \mathrm{O}$ & 1.17 & 0.281 & 21.67 & 2.6 & -11.42 & -11.78 \\
\hline 12 & $\left(\mathrm{CH}_{3}\right)_{2} \mathrm{O}$ & 1.29 & 0.192 & - & 1.4 & -8.76 & -8.48 \\
\hline 13 & $\mathrm{C}_{6} \mathrm{H}_{5} \mathrm{NH}_{2}$ & 1.53 & 0.382 & '24.03 & 28.0 & - & 19.58 \\
\hline 14 & $\mathrm{C}_{6} \mathrm{H}_{5} \mathrm{~N}\left(\mathrm{CH}_{3}\right)_{2}$ & 1.58 & - & 26.94 & 46.0 & - & 165.73 \\
\hline 15 & $\mathrm{C}_{5} \mathrm{H}_{5} \mathrm{~N}$ & 2.15 & 0.309 & 25.97 & 16.2 & - & 110.84 \\
\hline 16 & $\left(\mathrm{CH}_{3}\right)_{2} \mathrm{CHOH}$ & 2.95 & 0.299 & 25.37 & 4.3 & 99.06 & 102.52 \\
\hline 17 & $1,3-\left(\mathrm{NO}_{2}\right)_{2} \mathrm{C}_{6} \mathrm{H}_{4}$ & 3.74 & - & 17.06 & 86.1 & - & 605.34 \\
\hline 18 & $\mathrm{CH}_{3} \mathrm{CN}$ & 3.97 & 0.321 & 19.13 & 6.5 & 360.04 & 345.64 \\
\hline 19 & $\mathrm{C}_{6} \mathrm{H}_{5} \mathrm{NO}_{2}$ & 4.23 & - & 37.28 & 200.5 & 1332.06 & 1276.11 \\
\hline 20 & $1,2-\left(\mathrm{NO}_{2}\right)_{2} \mathrm{C}_{6} \mathrm{H}_{4}$ & 5.98 & 0.264 & - & 40.2 & - & 1286.22 \\
\hline
\end{tabular}

with $\omega=0.394$ dissolved in $n$ - $\mathrm{C}_{5} \mathrm{H}_{12}(\omega=0.351)$ and $\mathrm{C}_{6} \mathrm{H}_{6}(\omega=0.212)$ linearly correlate with the corresponding values of $Z_{\alpha}$. IMI energy grows $(1,2,8)$ with the increase in parameters $\mu, \alpha$, and $\gamma_{l}^{2}$ which determine electro-optical constants. IMI energy growth leads to larger errors in molar Kerr constants obtained within the limits of the orientational theory of $\mathrm{EB}$, which cannot fully account for all types of energy changes. $\Delta m K_{2 l}^{\mathrm{g}}$ is minimum for all substances dissolved in $n-\mathrm{C}_{7} \mathrm{H}_{16}$ and maximum for substances dissolved in $\mathrm{CS}_{2}$. The errors also increase with increasing electrical and polarizational characteristics of apolar and polar solutes dissolved in the same solvent: $\Delta m K_{2 l}^{\mathrm{g}}\left(\mathrm{CS}_{2}\right)<\ldots<\Delta m K_{2 l}^{\mathrm{g}}\left(\mathrm{C}_{14} \mathrm{H}_{10}\right)$ and $\Delta m K_{2 l}^{\mathrm{g}}\left(\mathrm{C}_{8} H_{10}\right)<\ldots<\Delta m K_{2 l}^{\mathrm{g}}\left(o-\mathrm{C}_{6} \mathrm{H}_{4}\left(\mathrm{NO}_{2}\right)_{2}\right)$ (Table III, Figs. 1 and 2$)$. Tangents $\operatorname{tg}\left(\varphi_{1}\right)$ of the linear relationship (8) are essentially equivalent to coefficients $B_{12}(I, \alpha, \mu)$ in (5) and correlate with magnitudes of molar Kerr constants of solutes. For instance, calculations of $\Delta m K_{2 l}^{\mathrm{g}}$ according to [19] resulted in $\operatorname{tg}\left(\varphi_{1}\right)=0.0271, m K^{\mathrm{g}}=17.16 \times 10^{12} \mathrm{CGS}$ units for $\mathrm{C}_{6} \mathrm{H}_{6}$ being the solute and $\operatorname{tg}\left(\varphi_{1}\right)=0.0558, m K^{\mathrm{g}}=10332.06 \times 10^{12} \mathrm{CGS}$ units for $\mathrm{C}_{6} \mathrm{H}_{5} \mathrm{NO}_{2}$ being the solute. 
(Units of tangents correspond to the units of coordinate axes of Figs. 1, 2.) Deviations from linearity between $m K_{2}^{l}$ and $Z_{\alpha}$ (and between $\Delta m K_{2 l}^{g}$ and $Z_{\alpha}$ ) in a series of apolar solvents indicate formation of hydrogen bonded and/or charge transfer complexes. The latter usually happens for solutes with significant polarizability anisotropies $\gamma_{l}^{2}$. The deviations can be explained by considering orientational dynamics of complexes and not of individual molecules. The deviations were found for solutions of $m, o-\mathrm{C}_{6} \mathrm{H}_{4}\left(\mathrm{NO}_{2}\right)_{2}, \mathrm{CH}_{3} \mathrm{CN}$, and $\mathrm{C}_{6} \mathrm{H}_{5} \mathrm{NO}_{2}$ in $\mathrm{C}_{6} \mathrm{H}_{6}$ ( $\pi-\pi^{*}$ type complexes) as well as for solutions of $\mathrm{CHCl}_{3}$ and $\mathrm{CS}_{2}$ in $\mathrm{C}_{4} \mathrm{H}_{8} \mathrm{O}_{2}$ (H-bond complexes) - Table III, Fig. 2. At the same time we observed no deviations for solutions with $\mathrm{CCl}_{4}$ and $\mathrm{CS}_{2}$ being the solvents. This observation proves non-local character of pairwise IMI involving the solvents and puts under doubt the propositions of [21] about H-bond complex formation between molecules of these solvents and proton donor solutes on one hand and about a significant role of interactions between group dipoles in dilute solutions based on these solvents on the other hand.

Molar Kerr constants of pure substances calculated from experimental data on electro-optical properties of binary solutions depend on a model for the internal field. A mere substitution of the Heaviside step function originally used by Onsager to describe electric induction on the molecular cavity continuum border for an exponent [20] notably decreased discrepancies $\left(\Delta m K_{2 l}^{\mathrm{g}}, l=1,2\right)$ between the experimental $\left(m K_{2}^{\mathrm{g}}\right)$ and calculated molar Kerr constants (Table III, Fig. 1, 2). Further refinement of the theory of EB by explicitly considering high and low frequency molecular polarizabilities, substituting Onsager cavity for a sphere filled with a solute "medium" and a point dipole in the center, and introducing an oscillating local electric induction function [2], leads to an improved description of electrostatic energy (5) of solute-solvent interaction. This, in turn, enabled us to carefully examine basic ideas of the theory of EB of condensed media and make its predictions more accurate [3]. Our studies on dielectric and electro-optical properties of dilute binary solutions presented here unambiguously evidence in favor of these modifications of the theory: $\Delta m K_{21}^{\mathrm{g}}>\Delta m K_{22}^{\mathrm{g}}>\Delta m K_{23}^{\mathrm{g}}$ and $\operatorname{tg} \varphi_{1}>\operatorname{tg} \varphi_{2}>\operatorname{tg} \varphi_{3}$ for all values of structural and physical-chemical properties of solutes, solvents, and solutions.

Relationship (8) was tested experimentally and found to be valid. The following argument substantiates its suggested practical application. A conceived weakening of IMI between a solute and a solvent, under the assumption of non-changing structural and physical characteristics of both, is asymptotically equivalent to transforming the solute into a gas. Hence, extrapolation of $\Delta m K_{2 l}^{\mathrm{g}}\left(Z_{\alpha}\right)$ to $Z_{\alpha} \rightarrow 0$ can be used as a method of evaluation of gas phase molar Kerr constants. Figures 1, 2 and Tables II, III show that the method can be applied only in conjunction with the new internal field model [2]. This model used in (3) to calculate the extrapolated values of molar Kerr constants $\infty\left(m K_{2 l}^{\mathrm{g}}\right)^{\mathrm{e}}$ gives small relative errors $\Delta m K_{l}^{\mathrm{e}}=\left|m K_{2}^{\mathrm{g}}-{ }_{\infty}\left(m K_{2 l}^{\mathrm{g}}\right)^{\mathrm{e}}\right| / m K_{2}^{\mathrm{g}}\left(\right.$ then $\left.\Delta m K_{3}^{\mathrm{e}} \approx 0\right)$. All other approaches predict significant repulsive interactions $\left(U_{\Sigma}>0\right)$ in the limit $Z_{\alpha}=0$. This unphysical situation cannot correspond to the gaseous phase of solutes. Relative errors in the extrapolated values of $\infty\left(m K_{2 l}^{\mathrm{g}}\right)^{\mathrm{e}}$ determined according to [19] $(l=1),[20]$ $(l=2)$, and [3] $(l=3)$ with respect to the corresponding experimental gaseous phase values $m K_{2}^{\mathrm{g}}$ form a series of inequalities $\Delta m K_{1}^{\mathrm{e}}>\Delta m K_{2}^{\mathrm{e}}>\Delta m K_{3}^{\mathrm{e}}$. Our 
吉

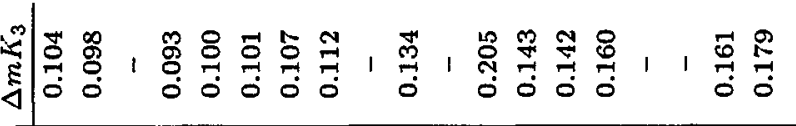

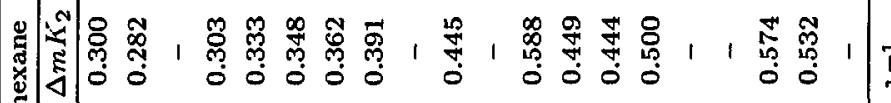

$\stackrel{\substack{0 \\ \frac{0}{2}}}{\frac{0}{2}}$ .

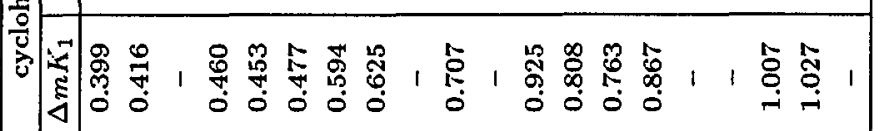

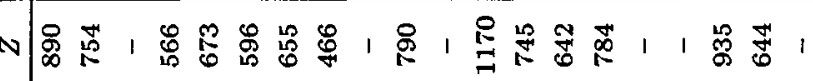

|

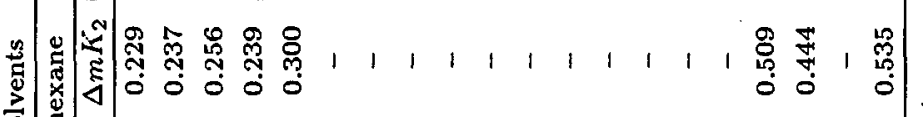

है

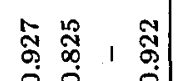

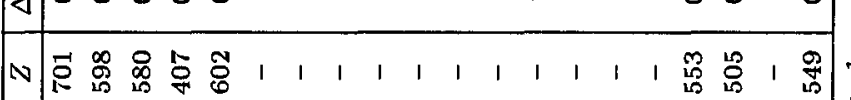

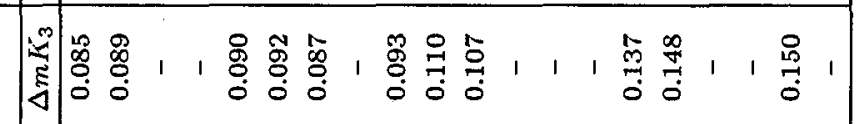

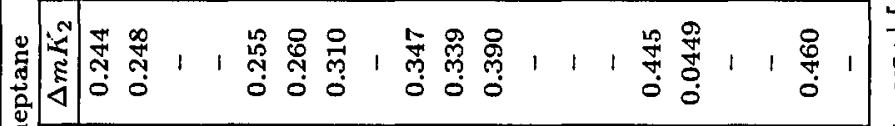

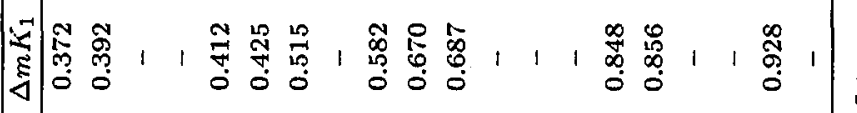

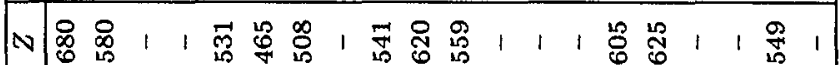

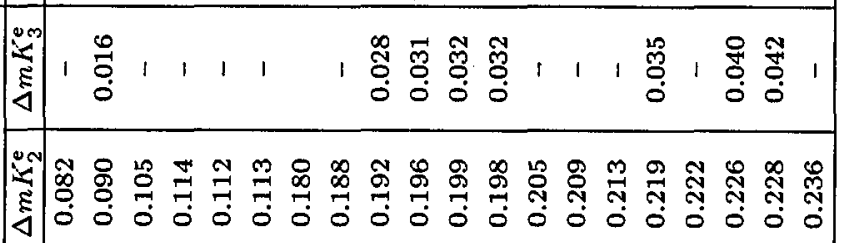

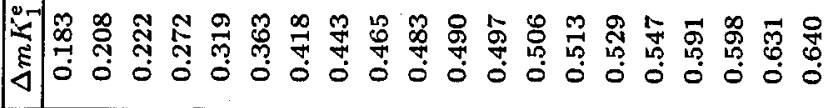

离

ర్⿻

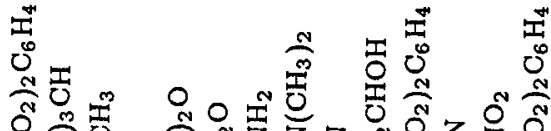




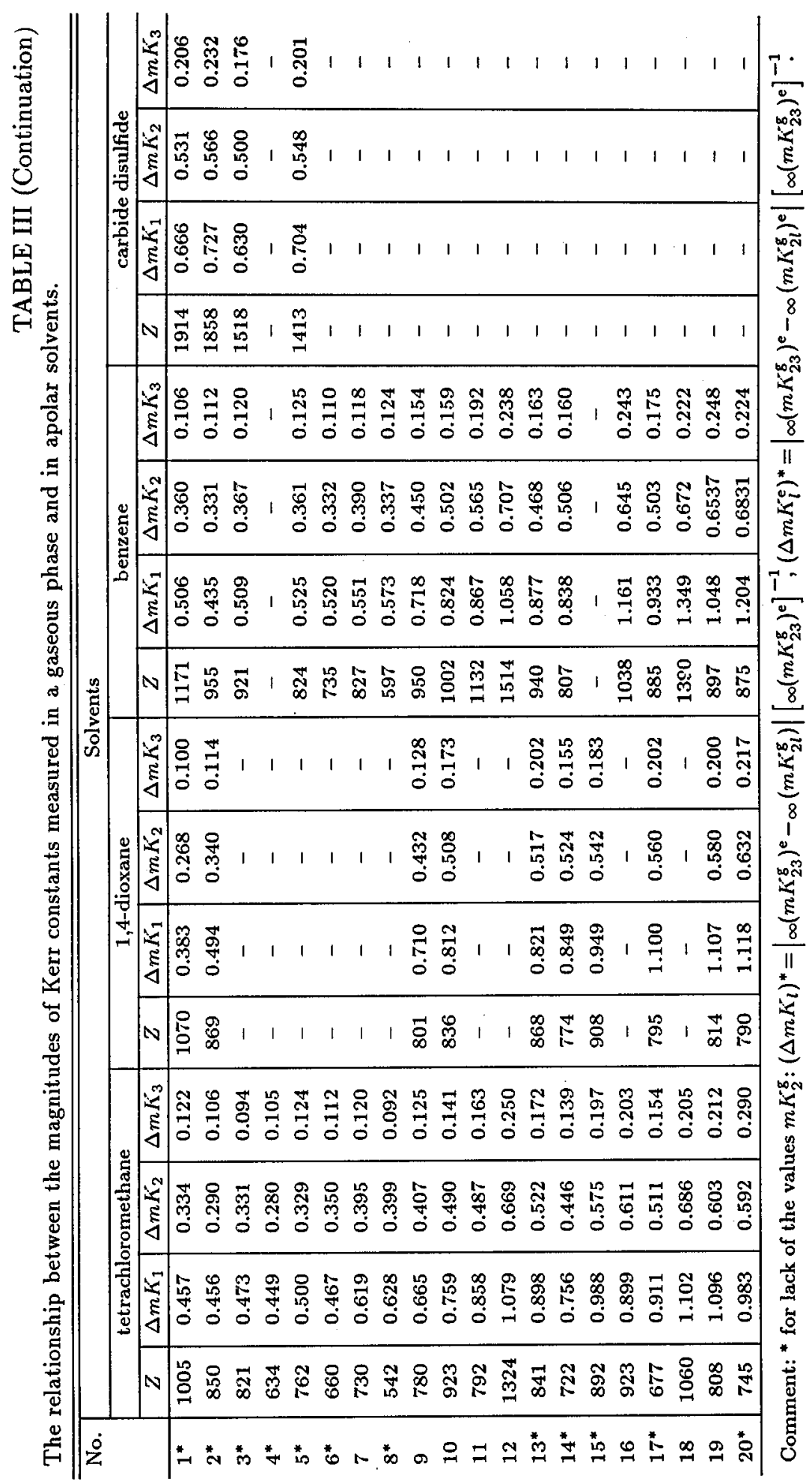


description [3], the only one that can be used to predict gas phase molar Kerr constants from experimental data on solutions, becomes extremely useful for the substances with limited solubility and for those which do not exist in gas phase.

\section{Conclusions}

This paper presented experimental results on electro-optical constants of dilute binary solutions characterized by a significant range of physical-chemical and structural properties. The results were used to test applicability of pairwise LD potentials to the description of potential energy of IMI in condensates. The effect of IMI on electro-optical characteristics of liquid media was investigated by studying correlations between the gas phase values of molar Kerr constants and those measured in dilute binary solutions. The following conclusions were made.

1. Molar Kerr constants of substances dissolved in a series of apolar solvents with molecules of similar sizes and first ionization potentials linearly correlate with $Z_{\alpha}=Z_{1} \alpha_{1} R_{1}^{-6}$. This characteristic of solutions was derived using LD potentials with the aim to describe the influence of dispersive and inductive pairwise interactions on electro-optical constants of condensates. The correlation holds only for substances which do not exhibit specific interactions.

2. Comparing various modifications of the orientational theory of EB and different models of internal field we concluded that the most promising were the approaches which develop the ansatz of reactive field, local dielectric permittivity, and quasi-continuum. The latter idea was introduced by substituting Heaviside step function used to describe electric induction on the Onsager cavity-continuum border for an oscillating distribution of electric induction in a sphere containing a solvated molecule of a solute in the middle. Such approaches were most successful in implicitly accounting for universal van-der-Waals interactions. This was evidenced by the minimal errors between the calculated and experimental gas phase molar Kerr constants.

3. An extrapolation method of evaluation of gas phase molar Kerr constants from experimental data on EB of dilute binary solutions was suggested. The method gave best results when applied in combination with the above mentioned modification of the internal field model and EB theory. The method was used to determine gas phase molar Kerr constants of twenty organic compounds most of which cannot be studied directly in gas phase.

\section{References}

[1] A.N. Vereshchagin, Polarizability of Molecules, Nauka, Moskva 1980, p. 177 (in Russian).

[2] N.I. Ivanov, O.V. Prezhdo, G.V. Tarasova, T.N. Kurskaja, V.V. Prezhdo, S.A. Tyurin, Zh. Fiz. Khim. (Russia) 68, 159 (1994).

[3] V.V. Prezhdo, G.V. Tarasova, O.V. Prezhdo, S.A. Tyurin, O.N. Akulova, T.N. Kurskaja, Acta Phys. Pol. A 85, 797 (1994).

[4] S.A. Tyurin, M.V. Khashchina, Zh. Fiz. Khim. (USSR) 61, 114 (1987).

[5] N.I. Ivanov, O.V. Prezhdo, G.V. Tarasova, T.N. Kurskaja, V.V. Prezhdo, S.A. Tyurin, Zh. Fiz. Khim. (Russia) 68, 912 (1994). 
[6] V.V. Prezhdo, M.V. Khashchina, V.A. Zamkov, Electro-optical Investigation in Physics and Chemistry, Vyshcha Shkola, Kharkov 1982, p. 152 (in Russian).

[7] R.S. Armstrong, M.J. Aronej, C.G. Le Fevre, R.J.W. Le Fevre, M.R. Smith, J. Chem. Soc. , 1474 (1958).

[8] J. Crossley, G. Williams, J. Chem. Soc. Faraday Trans. II 73, 1906 (1977).

[9] A.D. Buckingham, Trans. Faraday Soc. 77, 577 (1981).

[10] G.V. Tarasova, Effekt Kerra i dipolnyje momenty molekul zhidkikh dielektrikov. Avtoreferat diss. kand. khim. nauk, University of Kharkov, Kharkov 1991, p. 25 (in Russian).

[11] M.V. Volkenstejn, M.A. El'jashevich, B.N. Stepanov, Kolebania molekul, Vol. 2, GITL, Moskva 1949, p. 440 (in Russian).

[12] A.E. Luckij, B.A. Veretenchenko, I.S. Romodanov, V.V. Prezhdo, Zh. Strukt. Khim. 16, 686 (1975).

[13] L. Degreve, Mol. Phys. 40, 837 (1980).

[14] A.D. Buckingham, P. Stiles, G. Ritchie, Trans. Faraday Soc. 67, 577 (1971).

[15] A.N. Vereshchagin, Kharakterisliki anizolropii polarizujemosti molekul, Nauka, Moskva 1982, p. 308 (in Russian).

[16] V.V. Prezhdo, G.V. Tarasova, O.V. Prezhdo, S.A. Tyurin, O.N. Akulova, T.N. Kurskaja, Acta Phys. Pol. A 86, 327 (1994).

[17] R. Rid, Dj. Prausnic, T. Sherwood, Svojstva gazov $i$ zhidkostej, Khimija, Leningrad 1982, p. 592 (in Russian).

[18] F.I. Vilesov, Usp. Fotoniki, Izd. University of Leningrad, Leningrad 1969, p. 68.

[19] S. Filipczuk, G. Khanarian, J. Chem. Soc. Faraday Trans. 77, 477 (1981).

[20] H. Block, S.M. Walker, Chem. Phys. Lett. 19, 363 (1973).

[21] P. Suppan, Spectrochim. Acta A 28, 599 (1972). 